properties. Currently, two main strategies are used to solve the selectivity issue. The first is known as non-directed $\mathrm{C}-\mathrm{H}$ functionalization, and relies on the fact that the structure of a molecule confers slightly different properties on different $\mathrm{C}-\mathrm{H}$ bonds, thus enabling the preferential reaction of one hydrogen out of several others ${ }^{4,5}$.

The second strategy is called the directinggroup approach: a chemical group attached to a molecule acts as a hook that catches a catalyst and directs it to a specific $\mathrm{C}-\mathrm{H}$ bond ${ }^{6}$ (Fig. 1a). This strategy is widely used because it enables reactions that are often otherwise unachievable to be performed highly selectively. But it has a considerable drawback the directing group generally needs to be attached to the molecule in a separate, preliminary step, and then later removed. These additional steps lower the overall efficiency of the process and generate more waste than is formed in non-directed $\mathrm{C}-\mathrm{H}$ functionalizations. The non-directed approach is therefore more attractive, but also much more difficult to achieve, as shown by the scarcity of literature on this topic.

$\mathrm{C}-\mathrm{H}$ functionalization reactions of aromatic compounds (which contain benzene rings or their analogues) are potentially of great use for organic synthesis. Heteroaromatic compounds typically contain rings that incorporate nitrogen, sulfur or oxygen atoms, and are good substrates for non-directed $\mathrm{C}-\mathrm{H}$ functionalization. This is because the presence of those atoms within an aromatic structure allows the different hydrogen atoms on the ring to be easily discriminated.

By contrast, non-directed $\mathrm{C}-\mathrm{H}$ functionalization is much less established for simple aromatics (those that contain rings made up only of carbon atoms) ${ }^{7}$. Such substrates generally have poor reactivity, and the reactions, if they occur, are typically not selective for specific $\mathrm{C}-\mathrm{H}$ bonds - mixtures of several products are obtained. To overcome the first limitation, simple aromatics are usually used in much greater quantities than is suggested by the stoichiometry of the reaction. Indeed, they are commonly used as the solvent for the reaction. This greatly reduces the environmental and economic sustainability of the overall process.

Wang et al. propose a new solution to address the non-directed $\mathrm{C}-\mathrm{H}$ functionalization of simple aromatics. The authors discovered that the combination of a catalytic metal (palladium) with a particular ligand (a 2-pyridone; Fig. 1b) greatly alters the outcome of the transformation, compared to the outcome when no ligand is used. The ligand binds closely to the metal atom, which not only makes the resulting catalyst unusually reactive, but also protects it from degradation.

Remarkably, this 'super-reactive' palladium complex cleaves the $\mathrm{C}-\mathrm{H}$ bonds of various aromatic substrates, including electron-poor ones, which generally perform badly or fail in such reactions. Another fundamental advantage arising from the increased reactivity of this complex is that the aromatic substrates can be used as the limiting reagent ${ }^{8}$ (in lower molar quantities than the other reagents), rather than in much larger quantities. Moreover, the reactions produce rather high yields (60-80\%), limiting waste from the unconsumed substrate.

Notably, the binding of the ligand to the metal allows better recognition of the substrate by the resulting catalyst than that achieved by the 'naked' metal, resulting in improved (albeit still rather moderate) selectivity for one particular $\mathrm{C}-\mathrm{H}$ bond out of several others. Wang et al. demonstrate the practical utility of their reactions by using them to convert a few drug molecules and natural products into morecomplex structures. Such transformations are of interest to researchers in the pharmaceutical industry.

Some limitations still need to be overcome to realize the full potential of these reactions. In particular, achieving total selectivity for a particular $\mathrm{C}-\mathrm{H}$ bond is crucial. Ideally, the selectivity should be controlled completely by the catalyst so that substrates can be modified predictably, preferably at positions that are usually difficult to target. The reactions currently require a relatively large amount of catalyst, and the addition of a silver salt; if the amounts of these two compounds could be reduced, then the transformations would become even more attractive, particularly for industrial processes. Last, but not least, Wang et al. use their catalyst to install two types of chemical group onto substrates. If other groups could also be introduced, then the potential of the reactions would expand greatly.

This work represents an important step forward in catalytically modifying simple molecules without resorting to directing groups. By expanding the range of molecules that can undergo $\mathrm{C}-\mathrm{H}$ functionalization reactions, Wang and colleagues' strategy opens up fresh avenues for synthetic chemistry, and will no doubt rapidly become a useful tool for organic chemists. -

Joanna Wencel-Delord and Françoise Colobert are in the European School of Chemistry, Polymers and Materials, UMR 7509, University of Strasbourg, 67087 Strasbourg, France.

e-mail:wenceldelord@unistra.fr

1. Wang, P. et al. Nature 551, 489-493 (2017).

2. Gensch, T., Hopkinson, M. N., Glorius, F. \& Wencel-Delord, J. Chem. Soc. Rev. 45, 2900-2936 (2016).

3. Hartwig. J. F. J. Am. Chem. Soc. 138, 2-24 (2016).

4. Hartwig, J. F. \& Larsen, M. A. ACS Central Sci. 2, 281-292 (2016).

5. Kuhl, N., Hopkinson, M. N., Wencel-Delord, J. \& Glorius, F. Angew. Chem. Int. Edn 51, 10236-10254 (2012).

6. Chen, Z. et al. Org. Chem. Front. 2, 1107-1295 (2015).

7. Moritani, I. \& Fujiwara, Y. Tetrahedron Lett. 8 , 1119-1122 (1967)

8. Zhang, Y.-H., Shi, B.-F. \& Yu, J.-Q. J. Am. Chem. Soc. 131, 5072-5074 (2009).

\title{
Layered-up regulation in the developing brain
}

\begin{abstract}
Modification of messenger RNAs through a process called $\mathbf{m}^{6} \mathrm{~A}$ methylation facilitates dynamic temporal regulation of RNA levels in neural precursor cells, enabling fine-tuning of developing neuronal circuits in the brain.
\end{abstract}

\section{J. DAVID SWEATT}

$\mathrm{O}$ ne of the most highly evolved structures in the human brain is the cerebral cortex ${ }^{1}$, which contains precisely structured layers of neurons that are involved in many aspects of cognition, including information processing and memory storage. During brain development, both the birth of neurons and their time-dependent wiring into complex functional circuits that span multiple neuronal layers must be carefully regulated to ensure normal cortical function. However, the molecular mechanisms that underlie these developmental processes remain mysterious. Writing in Cell, Yoon et $a l^{2}$ report an analysis of gene regulation in the developing mouse cortex. Their work reveals a mechanism that mediates precise temporal control over gene expression to ensure proper function of neuronal precursors called radial glia.

The authors investigated a major player in gene regulation ${ }^{3-9}-$ the chemical attachment of a methyl group to a particular nitrogen atom of the nucleoside adenosine in messenger RNA. Such adenosine $N^{6}$-methylation (dubbed $\mathrm{m}^{6} \mathrm{~A}$ methylation) is catalysed in cells by Mettl 3 and Mettl14 enzymes, among others $^{7-9}$. 
Yoon et al. set out to determine the role of $\mathrm{m}^{6} \mathrm{~A}$ methylation in the nervous system. The authors analysed mice engineered either to lack the Mettl14 gene or to express lower than normal levels of Mettl3. They found that decreases in $\mathrm{m}^{6} \mathrm{~A}$ methylation caused by these mutations increased levels of various messenger RNA molecules. The researchers then demonstrated that RNA $\mathrm{m}^{6} \mathrm{~A}$ methylation causes enhanced RNA degradation by shortening the half-life of target mRNA species, limiting for how long the proteins that they encode can exert their effects in the cell. The affected transcripts mainly encode transcription factors and regulators of the cell cycle and neuronal differentiation. The authors found that, in wild-type embryos, RNA $\mathrm{m}^{6} \mathrm{~A}$ methylation led to coordinated downregulation of target transcripts during crucial phases of development, indicating that dynamic adenosine modification enables temporal fine-tuning of mRNA levels.

How does disrupting RNA $\mathrm{m}^{6} \mathrm{~A}$ methylation affect brain development? Yoon and colleagues showed that aberrant persistence of non-methylated transcripts in their mutant mice led to defects in the cell cycle in radial glia. These cells are not only neuronal precursors; they also act as scaffolds that can guide migration of newly formed neurons to the appropriate layer of the cerebral cortex ${ }^{10-12}$, ensuring their proper integration into functional circuits during development. The authors found that delays in radial-glia divisions led to the cells' abnormal persistence in the cortex after birth, and thereby disrupted cortical development. This caused disorganization of the finely layered architecture of the cortex, preventing normal circuit formation in cognition-related areas of the brain in mice.

Finally, the researchers extended their investigation to neuronal cells derived from human stem cells. They found that, as in mice, the development of human neuronal precursors is regulated by $\mathrm{m}^{6} \mathrm{~A}$ methylation. Indeed, some of the human mRNA transcripts regulated by this mechanism have previously been associated with human brain disorders.

Yoon and colleagues' findings together indicate that $\mathrm{m}^{6} \mathrm{~A}$ methylation is an 'ubercontroller' of mRNA stability across pathways that control the cell cycle and differentiation in radial glia. As a secondary consequence, this modification is a regulator of neurons. The work also opens up several avenues for future research. For instance, the researchers could not assess the cognitive consequences of the developmental disruption they observed, because mice died less than a month after birth. But this is an important area of potential investigation. Another question concerns whether the same mechanism might control the kinetics of decay in non-coding RNAs.

In addition, other roles for chemical modification of RNAs probably exist in the central nervous system (CNS), and it will

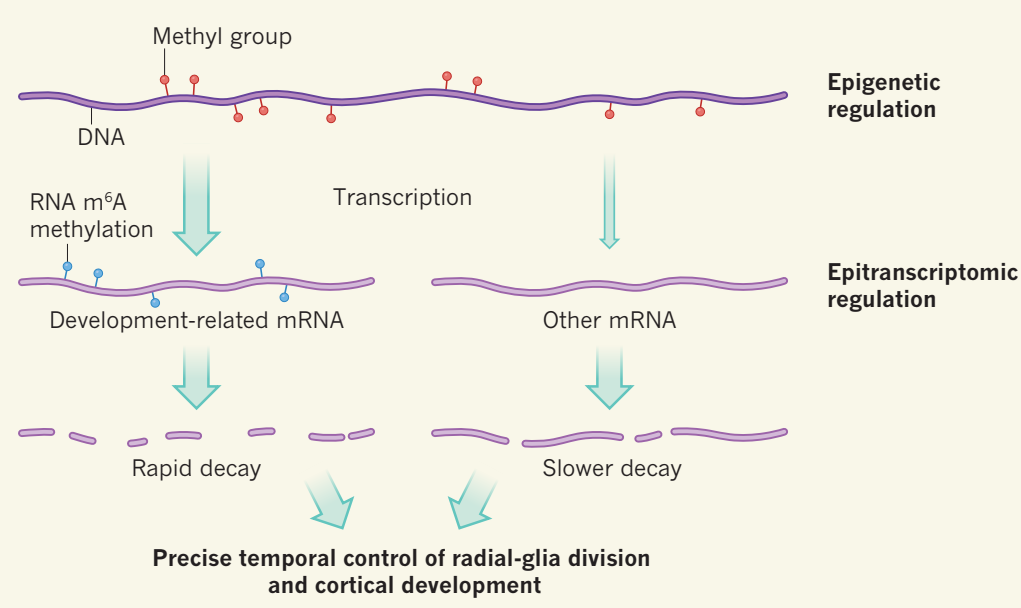

Figure 1 | Two layers of transcriptional regulation in the mouse cortex. The addition of methyl groups to DNA can lead to the modulation of gene-transcription levels (different levels indicated by size of arrow). Epigenetic regulatory mechanisms such as this, or chromosome packaging (not shown), which direct patterns of gene expression appropriate to a given cell type, are important in brain development ${ }^{12}$ Yoon et $\mathrm{al}^{2}{ }^{2}$ now demonstrate that development of the cortex of the embryonic mouse brain can also be regulated by the methylation of messenger RNA — specifically, of a nitrogen atom in the mRNA nucleoside adenosine. This $\mathrm{m}^{6} \mathrm{~A}$ methylation reduces the half-lives of mRNAs, causing rapid decay of those involved in developmental regulatory pathways in neuronal precursor cells called radial glia. Such regulation is essential for precisely timed cell divisions and differentiation of radial glia, and so for normal cortical development.

be worthwhile to dissect these. More than 100 forms of chemical modification of RNA are documented to exist. Among the most prevalent are the methylation of adenosine and of the base cytosine, and the catalytic conversion of the nucleoside uridine to an isomer called pseudouridine. However, their functional roles in the brain are poorly understood ${ }^{13}$. Nonetheless, with previous work indicating that $\mathrm{m}^{6} \mathrm{~A}$ methylation is a controller of cognitive function in the adult CNS, where it controls mRNA half-lives during the formation and storage of long-term memories ${ }^{14}$, there is a growing sense that a wide variety of roles for RNA chemical modification exists in the brain.

A major take-home message of Yoon and colleagues' work is that cortical development is under dual layers of control (Fig. 1). The first layer, previously well described ${ }^{12}$, involves methylation of DNA and the regulation of chromosome packaging - factors that modulate gene expression without altering the underlying DNA sequence. Such epigenetic regulation defines precise patterns of gene expression, regulated over time ${ }^{15}$. It is heritable over cell divisions because it relates to DNA, and has a role in maintaining cell identity over the lifespan of a cell lineage.

By contrast, the second layer of control, $\mathrm{m}^{6} \mathrm{~A}$ methylation, can be considered an epitranscriptomic mechanism - one involving chemical modifications that alter RNA properties. $\mathrm{m}^{6} \mathrm{~A}$ methylation controls the decay kinetics of individual transcripts and even of families of functionally related RNAs. In doing so, this mechanism allows finer temporal control of patterns of protein translation in individual cells as they undergo division and differentiation during key developmental time windows.

The ultimate output of this two-component epigenomic control system is highly refined kinetic control of the translational output of the genome at a given time. It will not be surprising if such a system is soon uncovered in many of the other organs that undergo complex structural development.

J. David Sweatt is in the Department of Pharmacology, Vanderbilt University, Nashville, Tennessee 37232, USA.

e-mail:david.sweatt@vanderbilt.edu

1. Herculano-Houzel, S., Manger, P. R. \& Kaas, J. H. Front. Neuroanat. 8, 77 (2014).

2. Yoon, K. et al. Cell 171, 877-889 (2017).

3. Li, X., Xiong, X. \& Yi, C. Nature Methods 14, 23-31 (2016).

4. Zhao, B. S., Roundtree, I. A. \& He, C. Nature Rev. Mol. Cell Biol. 18, 31-42 (2017).

5. Desrosiers, R. C., Friderici, K. H. \& Rottman, F. M Biochemistry 14, 4367-4374 (1975)

6. Meyer, K. D. \& Jaffrey, S. R. Nature Rev. Mol. Cell Biol. 15, 313-326 (2014).

7. Patil, D. P. et al. Nature 537, 369-373 (2016).

8. Wang, Y. et al. Nature Cell Biol. 16, 191-198 (2014)

9. Batista, P. J. et al. Cell Stem Cell 15, 707-719 (2014).

10.Noctor, S. C., Flint, A. C., Weissman, T. A., Dammerman, R. S. \& Kriegstein, A. R. Nature 409 714-720 (2001).

11.Breunig, J. J., Haydar, T. F. \& Rakic, P. Neuron 70, 614-625 (2011).

12. Taverna, E., Götz, M. \& Huttner, W. B. Annu. Rev. Cell Dev. Biol. 30, 465-502 (2014).

13. Leighton, L. J. et al. Genes Brain Behav. http://dx.doi. org/10.1111/gbb.12426 (2017)

14. Widagdo, J. et al. J. Neurosci. 36, 6771-6777 (2016).

15.Sweatt, J. D. Neuron 80, 624-632 (2013). 